\title{
World No Tobacco Day 2021 Impegnati a smettere!
}

\section{L'Organizzazione Mondiale della Sanità lancia una campagna per aiutare $\mathbf{1 0 0}$ milioni di persone che vogliono smettere di fumare e costituirà anche il tema della Giornata mondiale senza tabacco del 31 maggio 2021}

\author{
Paolo D'Argenio
}

La pandemia COVID-19 ha spinto milioni di consumatori di tabacco a pensare di voler smettere. La campagna sosterrà almeno 100 milioni di fumatori che cercano di smettere di fumare, attraverso la comunità di chi ha già smesso.

Nel mese di dicembre l'Organizzazione Mondiale della Sanità (OMS) ha avviato la campagna con: I'istituzione di un servizio che, tramite WhatsApp, aiuta chi vuole smettere di fumare, Quit Challenge [1] (la sfida di smettere), e la pubblicazione: More than 100 reasons to quit tobacco [2] (più di 100 ragioni per smettere).

\section{Florence: I'operatore digitale dell'OMS}

Un operatore sanitario digitale dell'OMS 24 ore su 24, 7 giorni su 7 per aiutare le persone a smettere di fumare, inizialmente disponibile solo in lingua inglese, è ora in grado di supportare le persone in arabo, cinese, francese, russo e spagnolo [3]. Impegnarsi a smettere aiuterà a creare ambienti più sani che favoriscono la cessazione del tabacco, e lo farà sostenendo politiche incisive a favore di chi smette di fumare, aumentando l'accesso ai servizi di cessazione, ampliando la consapevolezza delle tattiche dell'industria del tabacco e consentendo ai fumatori di fare tentativi di smettere

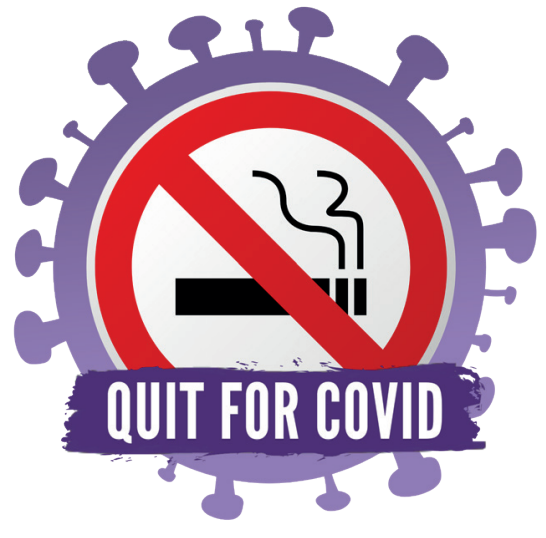

attraverso iniziative "Smetti e Vin$\mathrm{ci}^{\prime \prime}$, il programma dell'OMS attuato anche in Italia da alcune Asl.

L'OMS, insieme ai propri partner, creerà comunità digitali in cui le persone potranno trovare il supporto sociale di cui hanno bisogno per smettere. L'attenzione sarà rivolta ai Paesi ad alta prevalenza in cui vive la maggior parte dei consumatori di tabacco nel mondo.

Smettere di fumare è una sfida, ancor più oggi a causa dello stress sociale ed economico che è provocato dalla pandemia

In tutto il mondo circa 780 milioni di persone affermano di voler smettere, ma solo il 30\% di esse ha accesso ai trattamenti che possono aiutarle a farlo. Insieme ai partner, I'OMS fornirà alle persone gli strumenti e le risorse di cui hanno bisogno per tentare con successo di smettere.

"Il fumo uccide 8 milioni di persone all'anno, ma se ci fosse bisogno di una motivazione in più, la pandemia fornisce il giusto incentivo", ha detto il Direttore generale dell'OMS.

L'OMS ha pubblicato un documento scientifico [4] che mostra che i fumatori sono a maggior rischio di sviluppare malattie gravi e morte per COVID-19.

Il tabacco è anche un importante fattore di rischio per malattie non trasmissibili, come quelle cardiovascolari e quelle respiratorie, il cancro e il diabete. Inoltre, le persone con queste malattie sono più a rischio di sviluppare una forma grave di COVID-19.

L'OMS si appella ai Governi di tutto il mondo: forti servizi di cessazione migliorano la salute, salvano vite e fanno risparmiare denaro.

"Milioni di persone in tutto il mondo vogliono smettere di fumare: dobbiamo cogliere questa opportunità e investire in servizi per aiutarle ad avere successo, mentre esortiamo tutti a disinve- 
stire dall'industria del tabacco e dai loro interessi", ha affermato il dottor Ruediger Krech, Direttore della promozione della salute.

Per creare ambienti favorevoli all'abbandono del tabacco, I'OMS ha lavorato con partner e Paesi in tutto il mondo per implementare misure di controllo del tabacco che riducano efficacemente la do- manda di tabacco.

L'OMS chiede a tutti i Governi di garantire che i loro cittadini abbiano accesso a servizi per la cessazione: brevi consigli, linee telefoniche gratuite per smettere di fumare, servizi di messaggistica su telefono cellulare e operatori digitali per smettere, terapie sostitutive della nicotina e altri strumenti che hanno dimostrato di poter aiutare le persone a smettere di fumare.

[Tabaccologia 2021; XIX(1):11-12] https://doi.org/10.53127/tblg-2021-A003

\section{Paolo D'Argenio}

Editor di tobaccoendgame.it

Associazione Italiana di Epidemiologia (AIE)

\section{Bibliografia}

1. WhatsApp. Chatta su WhatsApp con World Health Organization. https://api. whatsapp.com/send/?phone $=41798931892 \&$ text=tobacco\&app_absent $=0$.

2. Tobacco endgame. Alleanza per un'Italia senza tabacco. Più di 100 motivi per smettere di fumare. 27 dicembre 2020. https://tobaccoendgame.it/convenzione/piu-di-100motivi-per-smettere-di-fumare/.
3. World Health Organization. Quit tobacco today! https:// www.who.int/news-room/spotlight/using-ai-to-quit-tobacco.

4. Tobacco endgame. Alleanza per un'ltalia senza tabacco. Organizzazione Mondiale della Sanità: Fumo e COVID-19. Sintesi dei dati scientifici. 27 luglio 2020. https://tobaccoendgame.it/news/organizzazione-mondiale-della-sanita-fumo-e-covid-19-sintesi-dei-dati-scientifici-al-30-giugno-2020/.

\section{OPEN ACCESS}

L'articolo è open access e divulgato sulla base della licenza CCBY-NC-ND (Creative Commons Attribuzione - Non commerciale - Non opere derivate 4.0 Internazionale). L'articolo può essere usato indicando la menzione di paternità adeguata e la licenza; solo a scopi non commerciali; solo in originale. Per ulteriori informazioni: https://creativecommons.org/licenses/by-nc-nd/4.0/deed.it 